\title{
Migratory large vessel vasculitis preceding acute myeloid leukemia: a case report
}

\author{
Dinusha Chandratilleke ${ }^{1,2^{*}}$ D, Anthea Anantharajah', Mauro Vicaretti ${ }^{3}$, Warwick Benson ${ }^{4}$ and Lucinda J. Berglund ${ }^{1,2}$
}

\begin{abstract}
Background: Large vessel vasculitis is a rare disorder usually occurring in the context of the autoimmune conditions of giant cell arteritis and Takayasu's arteritis. Case reports have described large vessel vasculitis occurring in individuals with myelodysplastic syndrome, preceding transformation to acute myeloid leukemia.

Case presentation: A 56-year-old Afghanistan-born woman presented with fever, a tender left carotid artery, and raised inflammatory markers. Computed tomography revealed thickening of the wall of her left carotid artery. Her symptoms resolved spontaneously; however, they recurred weeks later on the contralateral side, along with abdominal pain after eating. Further imaging with computed tomography and positron emission tomography demonstrated resolution of her left carotid artery abnormality, but new wall thickening and inflammation in her right carotid artery, abdominal aorta, and superior mesenteric artery. She was diagnosed as having large vessel vasculitis, which resolved with corticosteroids and methotrexate. Five months later, she developed acute myeloid leukemia. She had no known history of myelodysplastic syndrome at the time of diagnosis with vasculitis.
\end{abstract}

Conclusions: Large vessel vasculitis in older individuals presenting with atypical clinical features, such as a migratory pattern of affected vessels, vessel wall tenderness, and marked systemic inflammation, should prompt a search for underlying myelodysplasia. Clinicians should be vigilant for progression to acute myeloid leukemia.

Keywords: Large vessel, Vasculitis, Acute myeloid leukemia, AML, Autoimmune, Myelodysplasia, MDS, Migratory

\section{Background}

Large vessel vasculitis is a rare condition characterized by inflammation within the walls of the aorta and its major branches. It can occur in a range of autoimmune disorders, including Takayasu's arteritis (TA) and giant cell arteritis (GCA), and typically requires treatment with high doses of corticosteroids and other immunosuppressive agents. In the literature, there have been case reports of large vessel vasculitis occurring in individuals with myelodysplastic syndrome (MDS), some of whom have later developed acute myeloid leukemia (AML). We describe a patient with an atypical presentation of large vessel vasculitis, which was migratory in nature and accompanied by marked systemic inflammatory features. Unlike previous case reports, our patient did not have a prior diagnosis of MDS, but subsequently developed AML.

\footnotetext{
* Correspondence: cdinusha@gmail.com

${ }^{1}$ Department of Immunopathology, Pathology West, ICPMR, Westmead Hospital, Sydney 2145, NSW, Australia

${ }^{2}$ Faculty of Medicine, University of Sydney, Sydney, NSW 2000, Australia Full list of author information is available at the end of the article
}

\section{Case presentation}

A 56-year-old Afghanistan-born woman presented to our hospital with a 2-week history of constant left-sided neck pain, associated fevers $\left(38.0^{\circ} \mathrm{C}\right)$, and rigors. Her past medical history included mild asthma and osteoarthritis. A physical examination revealed exquisite tenderness over the anterior triangle of her left neck. Laboratory studies showed a white cell count of $4.1 \times 10^{9}$ cells/L (reference range 3.9 to 11.1 ), hemoglobin $112 \mathrm{~g} / \mathrm{L}$ (115 to 165$)$, mean corpuscular volume (MCV) $96 \mathrm{fL}$ (82 to 98), C-reactive protein (CRP) $109 \mathrm{mg} / \mathrm{L}(\leq 3)$, with normal electrolytes and liver function tests. Computed tomography (CT) of her neck (Fig. 1) revealed wall thickening of her left distal common carotid artery with hazing of the adjacent fat. Autoimmune serology, including anti-nuclear antibodies, anti-neutrophil cytoplasmic antibodies, antibodies to extractable nuclear antigens, double strandeddeoxyribonucleic acid (DNA) antibodies, rheumatoid factor, and antibodies to cyclic citrullinated peptide, were negative. These results were helpful in excluding a systemic small vessel vasculitis. Her serum complement 


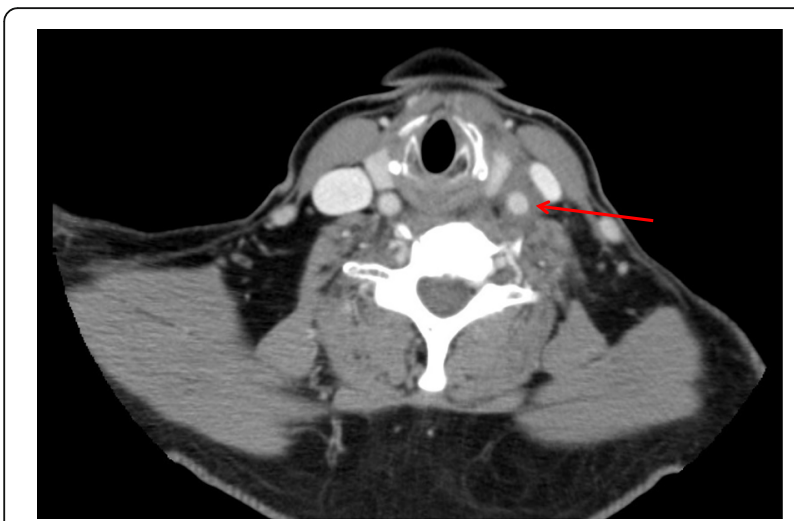

Fig. 1 Computed tomography of the neck on initial presentation revealing wall thickening of the left distal common carotid artery with hazing of the adjacent fat (red arrow)

levels were normal, and a left temporal artery biopsy was negative. Her neck pain and fevers resolved spontaneously without corticosteroids or other immunosuppression and she was discharged home on day 6. She presented 17 days later with fever $\left(38.0{ }^{\circ} \mathrm{C}\right)$ and abdominal pain 20 to $30 \mathrm{mi}$ nutes after eating. A CT of her abdomen (Fig. 2) revealed mild upper abdominal periaortic stranding with mural thickening, similar in appearance to her left common carotid artery from the original CT scan of her neck. During the next 24 to 48 hours, she developed new right-sided neck pain, contralateral to her pain on initial presentation. A CT angiogram revealed improvement in her left common carotid artery thickening, interval development of new right common carotid artery thickening, and hazing around her upper abdominal aorta, coeliac axis, and superior mesenteric arteries. A positron emission tomography (PET) scan

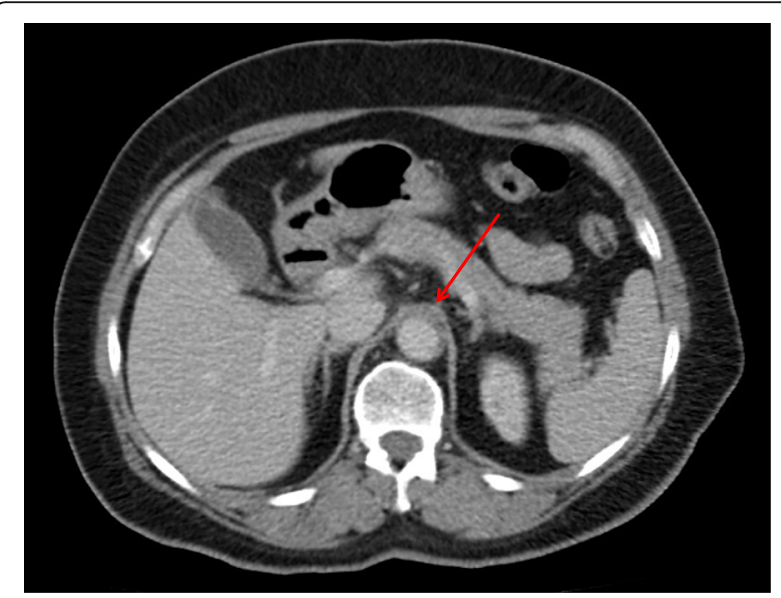

Fig. 2 Computed tomography of the abdomen on second presentation revealing mild upper abdominal periaortic stranding with mural thickening of the aorta (red arrow), similar in appearance to the left common carotid artery from the original computed tomography of her neck (Fig. 1)
(Fig. 3) demonstrated diffuse, intense fluorodeoxyglucose (FDG) uptake of her right common carotid artery, abdominal aorta, and superior mesenteric artery, consistent with a large vessel vasculitis, which had presented with a systemic inflammatory response, carotidynia, and mesenteric angina.

She was treated with methylprednisolone $(500 \mathrm{mg}$ daily for 3 days) administered intravenously with immediate resolution of her abdominal and neck pain and fevers. She was subsequently commenced on methotrexate $15 \mathrm{mg} /$ week administered orally and prednisone $50 \mathrm{mg} /$ day. She remained well over the following 5 months, with continuation of methotrexate, and her prednisone dose was gradually reduced to $15 \mathrm{mg} /$ day.

Five months later, she reported fatigue and persistent cough despite multiple courses of antibiotics. Blood tests revealed neutropenia with $>20 \%$ blasts on peripheral blood film. A subsequent bone marrow aspirate (Fig. 4) demonstrated a markedly hypercellular marrow with 69 $\%$ blasts, which by flow cytometry were identified as an abnormal population of myeloid cells expressing CD117, CD13, CD33, human leukocyte antigen-antigen D related (HLA-DR), CD123, lacking CD34, with aberrant CD4 expression, consistent with AML. Molecular studies showed a FLT3-internal tandem duplication (ITD) mutation, which characterizes more aggressive disease and frequent relapses [1]. She received induction chemotherapy with cytarabine and idarubicin but a month later a bone marrow aspirate demonstrated residual blasts $(9 \%)$ consistent with persistent AML. She received re-induction chemotherapy with cytarabine and idarubicin, and then further re-induction with fludarabine, cytarabine, and idarubicin. Despite initial morphological remission, her AML relapsed 3 months later. She had many infective complications, including febrile neutropenia and Clostridium difficile colitis, but her large vessel vasculitis did not recur. Due to a rapidly rising blast count and refractory disease, she was palliated and died 8 months after her diagnosis of AML.

\section{Discussion}

We present a case of large vessel vasculitis with atypical features, including a migratory involvement of affected vessels and marked systemic inflammatory features, with subsequent progression to AML.

Large vessel vasculitis associated with MDS has rarely been described [2]. In some cases, the diagnoses of MDS and large vessel vasculitis are made simultaneously, and in others, the MDS has been pre-existing. Our patient did not have a previous diagnosis of MDS before the development of vasculitis, and on presentation, had only minimally reduced hemoglobin $(112 \mathrm{~g} / \mathrm{L})$. Over the following weeks, her peripheral blood neutrophil count fluctuated between normal and mildly reduced ( 1.4 to $3.4 \times 10^{9}$ cells/ $\mathrm{L})$, as did her monocyte count $\left(0.5\right.$ to $1.8 \times 10^{9}$ cells $\left./ \mathrm{L}\right)$; 


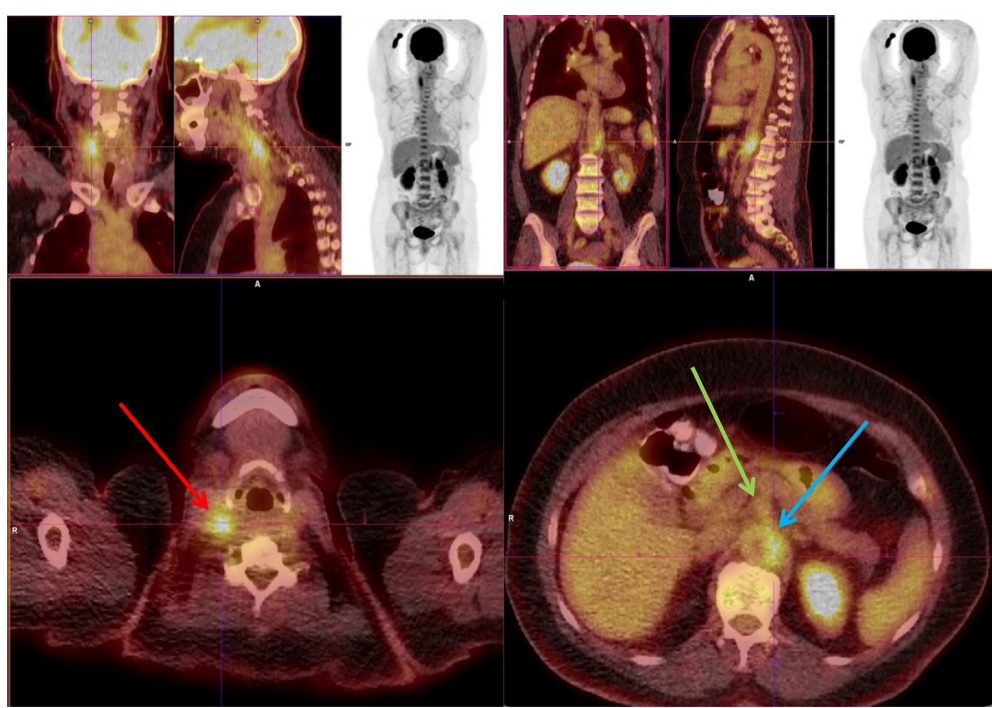

Fig. 3 Positron emission tomography scan demonstrating diffuse, intense fluorodeoxyglucose uptake of the right common carotid artery (red arrow), abdominal aorta (blue arrow), and superior mesenteric artery (green arrow)

however, her platelet count remained normal. With high dose glucocorticoids, her neutrophil count rose to $6.0 \times$ $10^{9}$ cells $/ \mathrm{L}$; however, the absence of a steroid-induced neutrophilia may also suggest a degree of myelodysplasia. The mild monocytosis, although not specific in the context of inflammation, may have been significant, given that her subsequent AML blasts aberrantly expressed CD4, which is normally expressed on monocytes.

Large vessel vasculitis can occur in autoimmune disorders, including extracranial GCA and TA. Marked systemic inflammation may be a feature of TA, however this disorder classically affects individuals younger than 50 years of age [3, 4]. GCA, more frequently seen in older individuals, typically involves smaller vessels such as the temporal arteries, but can also affect the aorta and its

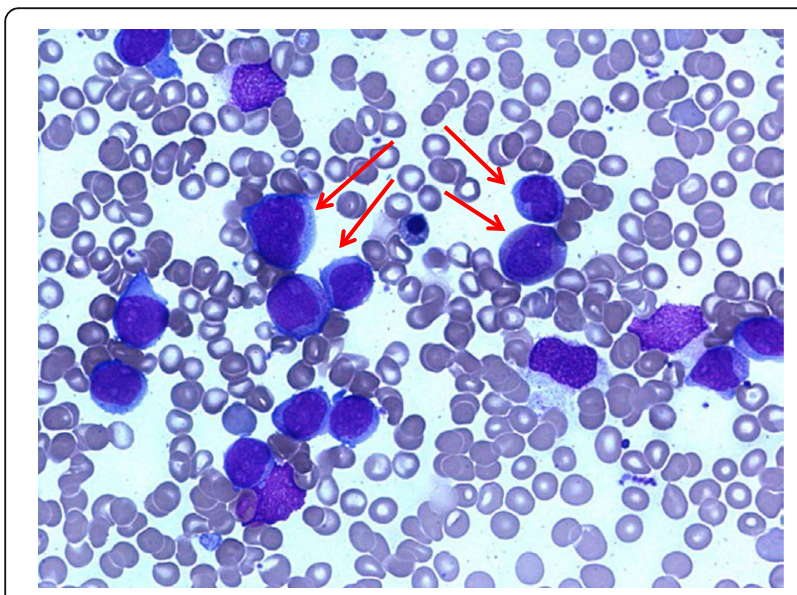

Fig. 4 Bone marrow aspirate demonstrating features of acute myeloid leukemia including myeloblasts (red arrows) $>20 \%$ major branches. The few reported cases of MDSassociated large vessel vasculitis presented in a similar manner to our patient: at an age $>50$ years, with acute inflammation, including tender vessels and a highly raised CRP $[2,5,6]$, exceeding the median CRP of $52 \mathrm{mg} / \mathrm{L}$ in patients with temporal artery biopsy-proven GCA [7].

In one review, five of eight patients with MDSassociated large vessel vasculitis developed AML, often refractory, usually within a year of presentation with vasculitis [2]. The timing of large vessel vasculitis preceding AML, and the unusual clinical features of acute inflammation with vessel wall tenderness and highly elevated inflammatory markers, suggest that the vasculitis in these patients may be a paraneoplastic phenomenon. The cytopenias in some forms of MDS may be immune mediated [8], with activated $\mathrm{T}$ cells inducing cytokine-mediated apoptosis of myeloid stem cells via tumor necrosis factor (TNF) and interferon gamma [5, 9]. Dysregulated immune mechanisms may thus be involved in the pathogenesis of MDS [10]. The association between MDS and autoimmune phenomena, such as arthritis, vasculitis, and connective tissue diseases, is well recognized in the literature [11, 12]; however MDS-associated vasculitis more commonly affects small caliber vessels [11, 13, 14].

Although the vasculitis in our patient responded promptly to glucocorticoids, the subsequent emergence of AML raises the possibility that immunosuppression impaired the cytotoxic anti-tumor response, and thus unmasked the AML. The majority of cases of patients reported with both large vessel vasculitis and MDS, however, received no steroid-sparing agent, yet several still progressed to AML [2]. In light of the poor prognosis when given corticosteroids, it is important to 
recognize the high risk of progression to AML in these individuals. Consideration may be given to simultaneous treatment for MDS, including azacitidine or even high intensity chemotherapy followed by allogeneic stem cell transplantation in selected patients with good performance status. A recent multicenter study confirmed that treatment with azacitidine in patients with both MDS and a systemic inflammatory or autoimmune disease appeared to have a positive effect on their inflammatory condition [15]; however, whether this treatment would reduce progression to AML in individuals with concurrent large vessel vasculitis is undetermined.

\section{Conclusions}

In summary, we present a 56-year-old woman with a large vessel vasculitis with migratory features, vessel wall tenderness, and marked systemic inflammation, who subsequently developed AML. Large vessel vasculitis with atypical clinical features in older individuals should prompt a search for underlying myelodysplasia, and vigilance for progression to AML.

\section{Abbreviations}

AML: Acute myeloid leukemia; CRP: C-reactive protein; CT: Computed tomography; DNA: deoxyribonucleic acid; FDG: Fluorodeoxyglucose; GCA: Giant cell arteritis; HLA-DR: Human leukocyte antigen-antigen D related; ITD: Internal tandem duplication; MCV: Mean corpuscular volume; MDS: Myelodysplastic syndrome; PET: Positron emission tomography; TA: Takayasu's arteritis; TNF: Tumor necrosis factor

\section{Acknowledgements}

We acknowledge Dr Maansi Joshi (Department of Haematology, Westmead Hospital, NSW Australia) for providing the bone marrow aspirate images.

\section{Funding}

None.

\section{Availability of data and materials}

Not applicable.

\section{Authors' contributions}

$D C, A A, W B, M V$, and $L J B$ were involved in patient care. $D C, A A$, and $L B$ wrote the manuscript. All authors read and approved the final manuscript.

\section{Competing interests}

The authors declare that they have no competing interests.

\section{Consent for publication}

Written informed consent was obtained from the patient's next-of-kin for publication of this case report and any accompanying images. A copy of the written consent is available for review by the Editor-in-Chief of this journal.

\section{Ethics approval and consent to participate}

Not applicable.

\section{Author details}

'Department of Immunopathology, Pathology West, ICPMR, Westmead Hospital, Sydney 2145, NSW, Australia. ${ }^{2}$ Faculty of Medicine, University of Sydney, Sydney, NSW 2000, Australia. ${ }^{3}$ Department of Vascular Surgery, Westmead Hospital, Sydney 2145, NSW, Australia. ${ }^{4}$ Department of Haematology, Westmead Hospital, Sydney 2145, NSW, Australia.
Received: 20 October 2016 Accepted: 12 February 2017

Published online: 16 March 2017

\section{References}

1. Small D. FLT3 mutations: biology and treatment. Hematology Am Soc Hematol Educ Program 2006:178-84. https://www.ncbi.nlm.nih.gov/ pubmed/17124058.

2. Katsuyama T, Uchida HA, Toma K, Maeda Y, Hirota D, Umebayashi R, Sada KE, Makino H. Large vessel vasculitis with myelodysplastic syndrome. Intern Med. 2014;53:63-6.

3. de Souza AW, de Carvalho JF. Diagnostic and classification criteria of Takayasu arteritis. J Autoimmun. 2014;48-49:79-83.

4. Freitas DS, Camargo CZ, Mariz HA, Arraes AE, de Souza AW. Takayasu arteritis: assessment of response to medical therapy based on clinical activity criteria and imaging techniques. Rheumatol Int. 2012:32:703-9.

5. Cohen MJ, Shyman A, Klein M, Ben-Yehuda A, Rubinow A, Or R, Goldschmidt N. Large vessel (Takayasu's) arteritis in a patient with myelodysplastic syndrome: is there a common pathogenesis? Clin Lymphoma Myeloma Leuk. 2011;11:60-3.

6. Steurer M, Fritsche G, Tzankov A, Gotwald T, Sturm W, Konwalinka G, Gruber J. Large-vessel arteritis and myelodysplastic syndrome: report of two cases. Eur J Haematol. 2004:73:128-33.

7. Kermani TA, Schmidt J, Crowson CS, Ytterberg SR, Hunder GG, Matteson EL, Warrington KJ. Utility of erythrocyte sedimentation rate and C-reactive protein for the diagnosis of giant cell arteritis. Semin Arthritis Rheum. 2012:41:866-71.

8. Okamoto T, Okada M, Mori A, Saheki K, Takatsuka H, Wada H, Tamura A, Fujimori Y, Takemoto Y, Kanamaru A, Kakishita E. Correlation between immunological abnormalities and prognosis in myelodysplastic syndrome patients. Int J Hematol. 1997;66:345-51.

9. Kochenderfer JN, Kobayashi S, Wieder ED, Su C, Molldrem JJ. Loss of T-lymphocyte clonal dominance in patients with myelodysplastic syndrome responsive to immunosuppression. Blood. 2002;100:3639-45.

10. Hamblin TJ. Immunological abnormalities in myelodysplastic syndromes. Semin Hematol. 1996:33:150-62.

11. Giannouli S, Voulgarelis M, Zintzaras E, Tzioufas AG, Moutsopoulos HM. Autoimmune phenomena in myelodysplastic syndromes: a 4-yr prospective study. Rheumatology (Oxford). 2004;43:626-32.

12. Tefferi A, Vardiman JW. Myelodysplastic syndromes. N Engl J Med. 2009;361:1872-85.

13. Castro M, Conn DL, Su WP, Garton JP. Rheumatic manifestations in myelodysplastic syndromes. J Rheumatol. 1991;18:721-7.

14. de Hollanda A, Beucher A, Henrion D, Ghali A, Lavigne C, Levesque $H_{\text {, }}$ Hamidou M, Subra JF, Ifrah N, Belizna C. Systemic and immune manifestations in myelodysplasia: a multicenter retrospective study. Arthritis Care Res (Hoboken). 2011;63:1188-94.

15. Fraison JB, Mekinian A, Grignano E, Kahn JE, Arlet JB, Decaux O, Denis G, Buchdahl AL, Omouri M, Maigne G, et al. Efficacy of Azacitidine in autoimmune and inflammatory disorders associated with myelodysplastic syndromes and chronic myelomonocytic leukemia. Leuk Res. 2016;43:13-7.

Submit your next manuscript to BioMed Central and we will help you at every step:

- We accept pre-submission inquiries

- Our selector tool helps you to find the most relevant journal

- We provide round the clock customer support

- Convenient online submission

- Thorough peer review

- Inclusion in PubMed and all major indexing services

- Maximum visibility for your research 